\title{
DOCUMENTATION AND HBIM OF INDUSTRIAL HERITAGE USING DRONE IMAGES: PETROLEUM RESERVOIR OF MASHHAD
}

\author{
R. Zaker ${ }^{1}$, A. Eghra ${ }^{2}$, P. Pahlavan ${ }^{3 *}$ \\ ${ }^{1}$ School of Architecture and Environmental Design, Iran University of Science and Technology, Iran \\ ${ }^{2}$ Persepolis World Heritage site, Iran \\ ${ }^{3}$ Faculty of Architecture and Urbanism, Ferdowsi University of Mashhad, Iran
}

KEYWORDS: Documentation; Industrial heritage; Digital twin; BIM; Drone photogrammetry.

\begin{abstract}
Documentation is a key step for cognition of cultural heritage, and also a requisite for conservation and adaptive reuse actions. Petroleum reservoir of Mashhad (constructed in 1925) was documented by means of Drone images. The photogrammetric documentation was aimed at provision of 3D models and as a dataset for the creation of BIM models. These data-enriched models could be used on Digital Twin platforms for monitoring and operational purposes, a concept that is becoming increasingly relevant in the field of cultural heritage conservation. The discussion of this process demonstrates that the dense matching of drone images may generate centimeter-level precision and can provide a proper basis for BIM and Digital Twin platforms. The capability of this system will help the community in sustainable development in order to preserve the monuments and determine the appropriate urban use in heritage buildings.
\end{abstract}

\section{INTRODUCTION}

Architectural heritage as a legacy of human beings from the past, incorporates a large spectrum of human values. The modern interpretation from cultural heritage $(\mathrm{CH})$, involves the built legacy with lives of people and has merged industrial heritage to the family of $\mathrm{CH}$ during the last decades. Industrial heritage elements, with their modern historical background, help the societies to pass on expressions to future generations. The modern documentation of architectural heritage involves various tools and techniques to fill the knowledge gaps. Modelling, management and conservation of modern heritage requires various systems such as photogrammetry, GIS, laser scanning, and Historic Building Information Modelling (HBIM) to argue for the necessity of cognition in $\mathrm{CH}$ interventions (Yang et al. 2020).

Petroleum reservoir of Mashhad dates back to a period in which petroleum was about to create a revolution in the economy of Middle Eastern countries (constructed in 1925). This three hectares site includes seven gigantic mazut storage tanks and several industrial sheds. One of the storage tanks with walls width of 9-12 $\mathrm{mm}$, benefitted the rivets system for steel sheets connections, as it dates back to a time in which welding technology was not imported to Iran. The site was documented by means of Drone images. The photogrammetric documentation was aimed at provision of $3 \mathrm{D}$ models and as a dataset for the creation of HBIM models.

BIM was originally used for building management purposes and assessments related to construction life-cycle issues. Meanwhile, as-built BIM and HBIM collaborate to the provision of various characteristics of built environment by reality-based data gain and concepts of reverse engineering (Arayici, 2008).

As-built HBIM is progressively utilized to manage and model valuable $\mathrm{CH}$ architectural elements by using photogrammetry and 3D point cloud from laser scanning and other reality-based recording data (Yang et al. 2020). The data-enriched models from the survey of the petroleum reservoir of Mashhad was assessed to be applied on Digital Twin platforms for monitoring and restorative purposes. This process explains that the dense matching of drone images can generate centimeter-level precision and can provide a proper basis for BIM and Digital Twin platforms. The potential for innovative application of the collected informative data is demonstrated and discussed in this short paper.

\section{NOVEL APPROACHES IN THE FIELD}

Documentation in the field of cultural heritage preservation is challenged by the complexity of the sources of information, by the need to integrate them in a holistic tool and by the way they are able to dialogue with society. (Van Balen, 2017). The Cultural Heritage Counts for Europe report (CHCfE, 2015) stresses the need for an integrated approach in which the social, cultural, environmental and economic impacts of heritage are fundamentally intertwined. This implies that a systematic approach in the creation, analysis and preservation of information related to the built environment, in this case cultural heritage, is desirable. Such information serves in the ongoing act of cultural preservation and can be shared with the community for further social and scientific purposes. 
The possibilities offered by today's tools and workflows for digital representation and information management open new perspectives in term of Data acquisition (Terrestrial Laser Scanning (TLS), Aerial \& Terrestrial Photogrammetry, Electronic Distance Measurement (EDM), etc.), data structuration (Computer Aided Design (CAD), Building Information Modeling (BIM), GIS (Geographic Information System), etc.), and data dissemination. The application of BIM principles to existing structures is made possible through the acquisition of high-quality point clouds obtained through TLS or photogrammetric surveys. On this basis, a digital model representing accurately buildings' present condition can be achieved and later on be used in a platform for collaborative data management. (Jouan et al. 2019)

This process, however, commences with a proper recording and documentation approach. Tools such as cartographic cameras, laser scanning, optical scanning, short-range systems (UAVs), GPS, manual and aerial laser scanning, and satellite images are some of the scientific and administrative tools for quantitative and qualitative optimization of project processes. In the following, the paper provides an executive report of the recording operation of the petroleum reservoir site of Mashhad, conducted using a UAV, a cartographic camera, a GPS, and an analog camera.

\section{RECORDING WITH A CARTOGRAPHIC APPROACH IN CULTURAL HERITAGE}

Recording is a prerequisite of documenting, conserving, restoring, reconstructing, and registering monuments and constructions. This document aims to record the visual condition of constructions or monuments, including damages, abrasions, deformations. This aim is often achieved by images, maps, sides, and reports. Several parameters should be carefully considered in recording monuments and historical and cultural sites: the ability to extract sufficient detail, digital recording capability, diversity of end products, and the possibility of using specific periods. In the documentation process, recording leads to the timely generation of information and related data in the form of drawings, photography, and so forth, allowing extracting information (descriptive geometrical) required in different applications.

It is possible to address the position, significance, and applications of recording on at least four levels: On the first level, recording is a sensible and useful package with independent value and utilization since recording a heritage construction or urban context allows further research and facilitates a comprehensive knowledge. In other words, it provides the raw material for various research types and functions as an introduction to the production of higher knowledge. It applies to teaching technical disciplines, including history, design, etc., and creating educational content on the second level. On the third level, it is necessary and useful for recording organizations, such as the Cultural Heritage Organization, responsible for registering the historical heritage and architecture.
On the fourth level, decision-making entities or city managers, such as the municipalities, can take advantage of it to both detect and tackle urban issues. Hence, the recording has a vital role in generating architectural knowledge and urban planning and has paramount applications in "education, research, media, policymaking, and technical content creation" (Noghrehkar, 2008).

Short-range photogrammetry has become increasingly popular as a data recording method in archeology. Short-range mapping allows for the creation of highly accurate three-dimensional models by imaging. This technique is commonly used for documentations with complex geometric properties or multiple spaces, including walls, pavements or sidewalks, rubble, and architectural components. Combining architectural information with information obtained from the recording of sites and monuments paves the way for ensuring the documentation or analysis of information (Optiz and Nolin, 1391: 21).

The conventional photogrammetry method involves analog photographs that create a so-called "three-dimensional image." It is used for stereo digitization and creating vector maps. This method is necessary to realize a set of geometric conditions, such as coverage and base-distance ratio, to ensure the accuracy needed and the possibility of a three-dimensional result. Relying on a combination of photogrammetric algorithms and computer vision, modern photogrammetric data processing is fully automated, allowing for implicit calibration of photographs and 3D reconstruction.

Photogrammetric modeling is an image-based method to create a 3-D model and extract quantitative and qualitative data. Portals et al. (2010) investigated the relationship between augmented reality and photogrammetry and used short-range photogrammetry and aerial photographs to develop a model for creating virtual reality. Emmett et al. (2010) proposed a method for 3-D modeling by combining short-range photogrammetry with aerial photographs to model small buildings, doors, windows, and some details not visible in aerial photographs. Finally, Yang et al. (2017) introduced a method to automatically create a 3-D model of constructions from aerial photographs and terrain mapping using an algorithm based on $\mathrm{C}++$.

The advantages of using UAVs include various advantages. The images taken by UAVs help create many views of terrestrial features, leading to a highly accurate model with numerous details. Modeling in UAVs is based on oblique imaging, making the selection of the proper shooting considerable.

\section{METHODOLOGY}

Recording operations were conducted on the macro and micro levels at the Mashhad petroleum reservoir site. On the macro level, the entire zone of the oil site with an area of $132678 \mathrm{~m} 2$ has was recorded using short-range photogrammetric tools (UAV), so the 
information collected allowed for creating accurate 3D models and maps of the entire study area. On the macro level, the intention was to describe and analyze the position and location of oil silos. The micro-recording was conducted using three approaches: 1 . surveying with Total Station and GPS (Shamim Iran), 2. Photogrammetry using UAVs and analog cameras by combining information provided by Total Station, and 3. Imaging using laser scanning. This recording method mainly focuses on the detailed plans of each oil silo and gives a complete description of the methodology and the resulting documentation. The work report was provided based on the performance of the recording and documentation team of Kohan Ghaleh South Co. The report involved using tools and instruments, the software required, and the output.

\section{MACRO-RECORDING}

The macro-recording of Mashhad petroleum reservoir site was conducted to recognize the map and coordinates of the area using short-range photogrammetric tools (UAVs). In this operation, first, the passages and spaces of the area were marked along with the mass and the interior space of the area and harvested using GPS in Iran's Coordinate Network (Shamim). The purpose of marking and coordinating markers using GPS tools is to use them as baseline points to improve the coordinate quality of the information in the Universal Transverse Mercator (UTM).

In the project area, 8 points on the interior spaces of the site and 18 base points (markers) were located in the entire area and extracted using GPS. The GPS used in this project was i-Target AT70 and Raymand iRoPro-G3B under Shamim.

The collected information was matched with the defined range after creating and removing the base points to identify and formulate UAV flight routes for photogrammetry. As depicted in Fig. 5, in recording the macro area (area 28 of oil site 1), the following parameters were selected: flight height of 70 meters and the image overlap of $75 \%$ longitudinal and $70 \%$ transverse with a 75 -degree angle of shoot in the grid capture mode. Grid capture was selected to create the 3-D effect, allowing for displaying the study area as a mobile space on the web (Fig. 1)

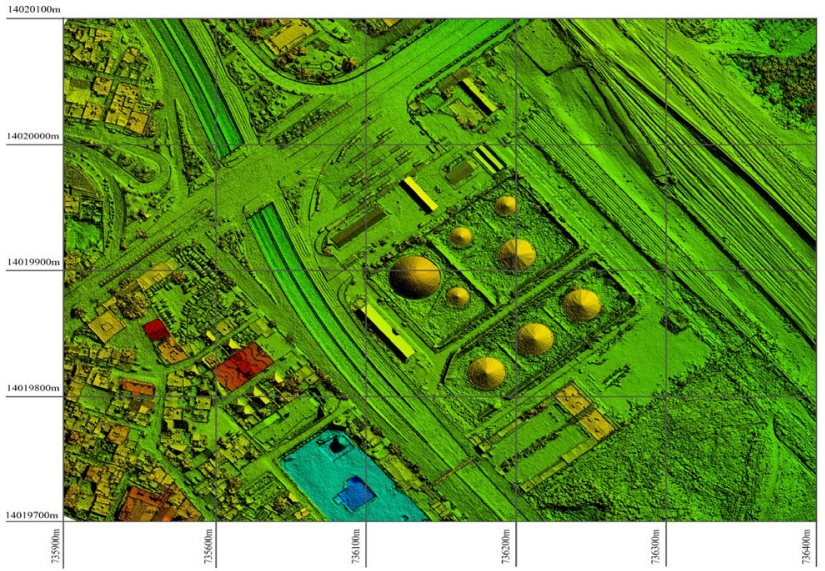

Fig. 1. 3-D capture by UAV

\section{DATA PROCESSING}

The collected data from the UAV and GPS (Shamim) tools were processed with photogrammetric engineering applications, such as Agisoft, 3DSurvey, and Context Capture, to prepare and create a map. In this paper, the processing performance of Agisoft Metashape has been reported. To this end, first, the image information captured by the UAV in photogrammetric operations is entered into Metashape.

After importing the images taken by the UAV into the Metashape, the images should be converted to sparse point cloud to process which the Align Photos option is selected. Creating sparse point cloud helps integrate the collected images and convert the Lat \& Long image coordinate system to UTM. Note that the UAV used in the Iranshahr Shiraz project was equipped with the GPS-RTK, ensuring the images' accurate coordinates.

The base points (markers) extracted by GPS should be matched to sparse point cloud to improve the numerical accuracy of the project from $7 \mathrm{~cm}$ to less than $2 \mathrm{~cm}$. The Reference shortcut deals with this in Metashape.

Increasing the numerical accuracy of the project ensures the processing can continue to the point that the map is created with complete confidence in the information collected. The sparse point cloud should be converted to dense point cloud to output an integrated image (Orthophoto) and elevation models (DEM, DSM) and continue processing.

After processing the dense point cloud, the Build DEM option in the Workflow shortcut can be used to process the elevation model (DEM). Then, the Build Orthomosaic menu enables processing the Orthophoto. 
Meta shape was used for processing the image information obtained from photogrammetry. The following information is output and transferred to Global Mapper, Civil3d, and GIS to generate the map. The steps are described as follows.

1) Ortho-mosaic

2) DEM

3) DSM

4) Point Cloud (Dense)

5) 3D Model

6) Reporter

7) Analog images of the study area

\section{THE MAPS GENERATED IN GLOBAL MAPPER}

To generate topography using Global Mapper, the DEM elevation model can be imported into it. Then, using the Create Contours command, it is possible to prepare the topography with the desired level curve for transfer to Civil3D. One of the most practical features of Global Mapper is that it can recognize different formats for converting and analyzing mapping information.

\section{THE MAPS GENERATED IN CIVIL3D}

Civil3D is an interdisciplinary engineering software with multiple capabilities in surveying, urban planning, and civil engineering. Civil3D can be used to generate UTM maps and metric zoning. Using Ortho-mosaic, Benchmark, and topographic outputs provide accurate information.

\section{THE MAPS GENERATED IN GIS}

GIS is a descriptive and analytical software with a very strong background in storing and displaying geographical and spatial data. GIS is an official analyst displaying solid engineering performance in information management. In this paper, GIS was selected to be the basis for monitoring project information. Following the defined purpose for monitoring, the executive team prepared a suitable context and the proper feed for monitoring.

\section{MAP INFORMATION REGARDING MACRO- RECORDING}

Macro-recording aimed to systematically identify the project area, obtaining accurate quantitative and qualitative data using novel instruments. Based on the work report, the recording and documentation team of Ferdowsi University attempted to create a smooth process based on the following diagram and designating an executive chart with distinct roles. (Table 1)

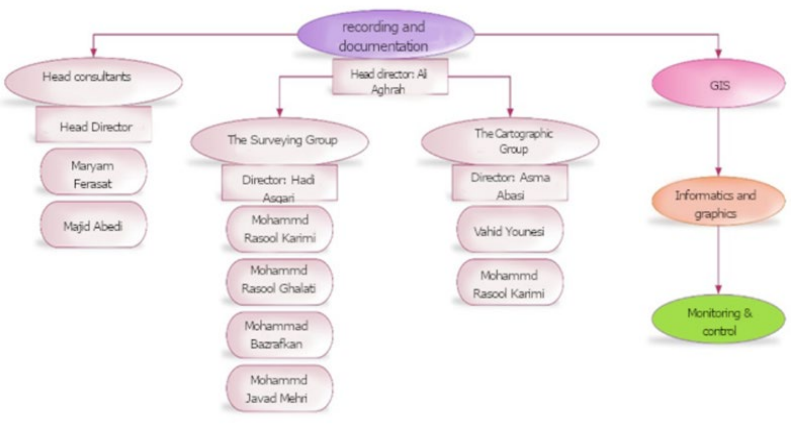

Table 1. Roles and Teams diagram

\section{MACRO-RECORDING MAPS}

As explained, the following data and maps were obtained using the adopted approach and related engineering software.

\section{MICRO-RECORDING}

The recording team mainly focused on the micro-section of the oil site in Mashhad. Micro-recording intended to prepare a systematic plan of the current state of silos and spaces and identify proximities with the metric approach of recognizing the state of passages, locating spaces, identifying and recognizing current use, and morphological status. The conducted micro-recording involved ground surveying with the Total Station Camera, photogrammetry with laser scanning devices, and color photography. The micro and macro recording data were combined to integrate micro-recording. Accordingly, some of the historical houses considered in microrecording were captured separately with a UAV at the height of 50 meters, and the collected data were processed.

\section{SYSTEMATIC GROUND MAPPING WITH THE TOTAL STATION CAMERA}

The main purpose of map surveying is to pinpoint the relative position of points on or near the ground surface. This simple definition implies that the goal is to determine the 3-D coordinates of points. Obviously, in some cases, the time dimension is also considered for this purpose, for instance, in astronomical measurements and satellite mapping. The mapping operation often comprises two stages: extracting or measuring and calculating and presenting the Cartesian work results. Various methods, devices, and instruments are used to obtain the necessary data for the second stage. In all methods, the errors are first investigated, and if acceptable, they are adjusted. Finally, the results are presented in analog form (map). Selecting digital (tables, digital models of the 
earth) and appropriate methods is a function of the size of the area, the desired accuracy, and the available facilities. The effect of the earth's sphericity is almost negligible in mapping small areas, and the earth can be considered flat in a small area. In other words, the base levels perpendicular to the length of the plummet are parallel and will be parallel at different points if, in fact (assuming the earth is spherical), the verticals at different points are not parallel to the plane and pass through the center of the earth. When the earth is assumed flat, the procedure is called plane surveying. These assumptions are true as long as the desired area does not exceed a few hundred square kilometers. Plane surveying is used in architectural engineering, urban planning, archeology, real estate, commercial and exploratory operations.

In archeology, surveying records old buildings and artifacts plans and assists in preparing detailed maps of restoration operations of facades, intersections, and rows, often to renovate demolished buildings. Map preparation includes two global stages: ground operations and office work. Ground operations involve preliminary identification of the area of operations and conducting the measurements required to determine the lengths, angles, and similar parameters, recording measurements in special drafts and forms. Office work deals with preparatory calculations to implement the measurements on the map.

The main model adopted by the recoding and documentation team in capturing historic houses using the Total Station camera allows for creating a systematic, scaled, 3-D cartographic model of the desired construction in engineering software. Civil3d and Rhino were used for this purpose.

The surveying team used Leica's TS02 Total Station Camera and Shamim's GPS device. The surveying operation was totally systematic and metric in the UTM.

\section{Photogrammetry with a laser scanner}

The laser scanner can capture millions of points in a remarkably short time, providing high speed in locating points and minimizing the volume of field operations. Furthermore, the high point density gives this device the accuracy that makes it suitable for modeling and recording historical monuments and highly detailed modeling. However, the main drawbacks of this device are high cost, relatively high weight, and many hidden areas.

In the site of Mashhad petroleum reservoir, the idea was to collect valuable information from a distinct silo. The first method was using point cloud with a laser scanner device, and the second method involved photogrammetry with UAVs and color photography. This operation aimed to protect the existing information of the intended houses and conserve it for the future. Moreover, this method's high accuracy makes it possible to obtain accurate architecture maps for further analysis. The final output required for the next phase of the project is a point cloud file (Fig.
2) with a suitable format which will be discussed in the next sections.

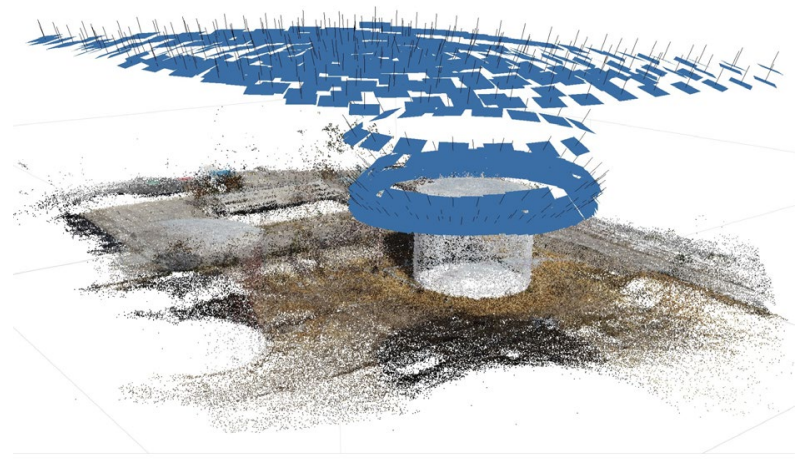

Figure 2. Point Cloud produced by UAV images

\section{BIM MODEL OF THE PETROLEUM RESERVOIR OF MASHHAD}

Building information modeling (BIM) has received significant research attention in the

field of built heritage. As-built BIM refers to a BIM representation of the "as-is" conditions of built heritage at the time of a survey. (Sun et al. 2019). Recent research works have increasingly mentioned this process and the resulted 3D model as Heritage BIM or simply HBIM. The need for 3D visualization in Geographic Information System (GIS) to enable better

visualization and analysis of complex issues related to elements of significance has led researchers in the field to progressively consider HBIM as a valid and practical approach. (Jouan et al. 2020)

This process involves three main stages; data acquisition, preprocessing of survey data and a modelling stage. (Dore et al. 2017). In the previous section, the first two stages were extensively discussed. 3D modelling from remotely sensed survey data is still, however, a manual and long process with much demand for new automated solutions. One area where progress is being made towards automated as-built BIM is in automated object recognition and feature extraction from point clouds. This refers to for example automatically detecting windows or doors from an image or point cloud (Zhang et al. 2014).

The processed data from the recoding and documentation works could be exported in various formats. For the accurate modelling of the captured data, a Point Cloud file format is suitable to be used as the reference. As we chose Revit software for the creation of our BIM models, we needed to provide a file format compatible with this software. Revit recognizes the Point Cloud Projects (*.RCP) and Point Cloud (*.RCS), both native Autodesk formats. 
Therefore, it was necessary to convert other point cloud formats to either of these two. The Autodesk Recap software was used to make this conversion and eventually the point cloud was imported in Revit software. (Fig.3)

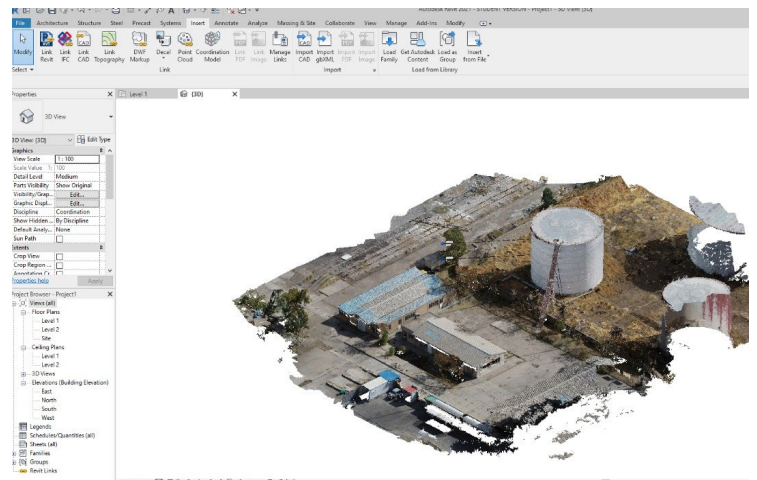

Figure 3. Point Cloud of the site imported in Revit

The point cloud file provides an accurate geometrical basis for the modeling process, indicating the location of various building and their elements on the site. Using the conventional modeling tools in Revit, it is possible to build up the model with precise geometrical data provided by the point cloud. In order to enrich the model with data for further purposes such as its employment on Digital Twin platforms, the building elements such as walls and roofs were defined with accurate data related to their materials and dimensions.

Regardless of the method of the 3D model creation, quality control of the final model is a crucial part of HBIM process. This is particularly important when BIM is used to produce documentation for conservation work, restorations, retrofitting or performing different types of building analysis. This can be manually carried out by playing the point cloud visualization and the final model and looking for differentiations. Upon the completion of the modeling and quality control processes, the data enriched model is ready for various monitoring and conservation approaches.

\section{DIGITAL TWINS AND HBIM}

The Digital Twins (DT) concept, originally defined as a "virtual representation of what has been produced" (Grieves et al. 2016) has gradually attracted the attention of the built environment experts. A digital copy of the physical assets connected to knowledge databases as well as sensors providing real-time operational data from the real world. This system permits the constant monitoring of the assets and improving its performance by analyses of the data acquired from the on-site sensors. The as-built BIM model provides an adequate basis for the knowledge database and visual representation of the assets. In this work, the BIM model of the site was prepared with requirements to be placed on a DT platform such as Smartspaces.io. The future research work would be focused on equipping the petroleum reservoir site with sensors and deploying the DT concept. Then the system would be analyzed and evaluated to determine its practicality for the desired conservation purposes.

\section{CONCLUSIONS}

Documentation and HBIM of Industrial Heritage Using Drone Images brings a large spectrum of potentials for cognition, conservation and adaptive reuse of built environment. This method of documentation on the case study of petroleum reservoir of Mashhad, was aimed at provision of 3D models and as a dataset for the creation of BIM models. The opportunity of using these dataenriched models to be used on Digital Twin platforms for monitoring and operational purposes was explored.

The data-enriched models from the survey of the petroleum reservoir of Mashhad was assessed to be applied on Digital Twin platforms for monitoring, maintenance, conservation and refurbishment purposes. This process explains that the dense matching of drone images can generate centimeter-level precision and bring a potential to provide a proper basis for BIM and Digital Twin platforms.

The point cloud file provided an accurate geometrical basis for the modeling process, indicating the location of various buildings and their elements on the site. Using the conventional modeling tools in Revit the model with precise geometrical data was built. In order to enrich the model with data for further purposes such as its employment on Digital Twin platforms, the building elements were defined. The as-built BIM model provides an adequate basis for the knowledge database and visual representation of the assets. However, future research works are required to be focused on equipping the industrial heritage sites with sensors and deploying the Digital Twins concept.

The quality control of the final can be carried out by playing the point cloud visualization and the final model and looking for differentiations. Upon the completion of the modeling and quality control processes, the data enriched model is ready for various motivations. The capability of this system can be used in order to preserve the monuments and determine the appropriate actions for the heritage buildings.

\section{REFERENCES}

Arayici, Y., Towards building information modelling for existing structures, Struct. Surv. 26 (2008) 210-222.

Yang, X., Grussenmeyer, P., Koehl, M., Macher, H., Murtiyoso, A., \&amp; Landes, T. (2020). Review of built heritage modelling: Integration of HBIM and other information techniques. Journal of Cultural Heritage, 46, 350-360.

Cultural Heritage Counts for Europe, 2015. CHCfE Consortium. 2015. Retrieved from: http://bit.ly/2jERIwx. As stated in article 
3.22 of (ICOMOS, 2003): all the activities of checking and monitoring should be documented and kept as part of the history of the structure.

Dore, C. and Murphy, M.: Current state of the art historic building information modelling, Int. Arch. Photogramm. Remote Sens. Spatial Inf. Sci., XLII-2/W5, 185-192, https://doi.org/10.5194/isprs-archives-XLII-2-W5-185-2017, 2017.

Grieves, M., Vickers, J., 2016. Digital twin: mitigating unpredictable, undesirable emergent behavior in complex systems. In Kahlen, F.J., Flumerfelt, S., Alves, A., Transdisciplinary Perspectives on Complex Systems: New Findings and Approaches, 85-113. doi.org/10.1007/978-3-319-38756-7_4.

Jouan, P. and Hallot, P.: Digital twin: a hbim-based methodology to support preventive conservation of historic assets through heritage significance awareness, Int. Arch. Photogramm. Remote Sens. Spatial Inf. Sci., XLII-2/W15, 609-615, https://doi.org/10.5194/isprs-archives-XLII-2-W15-609-2019, 2019.

Sun, Zheng; Xie, Jiangtao; Zhang, Yingying; Cao, Yongkang. 2019. "As-built bim for a fifteenth-century chinese brick structure at various lods" ISPRS Int. J. Geo-Inf. 8, no. 12: 577. https://doi.org/10.3390/ijgi8120577

Van Balen, K., 2017. Challenges that preventive conservation poses to the cultural heritage documentation field. Int. Arch. Photogramm. Remote Sens. Spatial Inf. Sci., XLII-2/W5, 713717.doi.org/10.5194/isprs-archives-XLII-2-W5-713-2017.
Yang, X., Grussenmeyer, P., Koehl, M., Macher, H., Murtiyoso, A., \&amp; Landes, T. (2020). Review of built heritage modelling: Integration of HBIM and other information techniques. Journal of Cultural Heritage, 46, 350-360.

Zyari, K., 2003, The impact of culture in the city (emphasis on islamic culture), Geography and Development Iranian Journal, No 2, PP. 95-109. (In Persian) 20's District of Tehran Municipality, http://region20.tehran.ir.

Zhang, R. and Zakhor, A. 'Automatic identification of window regions on indoor point clouds using LiDAR and cameras'. Applications of Computer Vision (WACV), 2014 IEEE Winter Conference on, 24-26 March 2014, 107-114. 